\title{
Systematic Study of the Glutathione (GSH) Reactivity of N- Phenylacrylamides: 2. Effects of Acrylamide Substitution
}

Adam Birkholz*, David J. Kopecky, Laurie P. Volak, Michael D. Bartberger, Yuping Chen, Christopher M. Tegley, Tara Arvedson, John D. McCarter, Christopher Fotsch, and Victor J. Cee

Departments of Medicinal Chemistry, Pharmacokinetics and Drug Metabolism, Molecular Structure, Discovery Technologies, and Oncology Research, Amgen Inc., One Amgen Center Drive, Thousand Oaks, California 91320, United States

\section{Supporting Information - Table of Contents}

\section{Additional Computational Discussion}

A. Full Citations

B. Validation: Simple substituents at $\alpha$ - and $\beta$ - positions.............................................S1

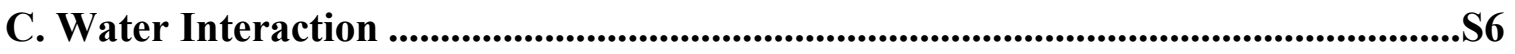

D. Additional Figures . $\mathrm{S10}$

\section{Computational Data}

\section{A. Full Citations}

All DFT calculations were carried out using G16 Rev C.01. Full reference:

Gaussian 16, Revision C.01, M. J. Frisch, G. W. Trucks, H. B. Schlegel, G. E. Scuseria, M. A. Robb, J. R. Cheeseman, G. Scalmani, V. Barone, G. A. Petersson, H. Nakatsuji, X. Li, M. Caricato, A. V. Marenich, J. Bloino, B. G. Janesko, R. Gomperts, B. Mennucci, H. P. Hratchian, J. V. Ortiz, A. F. Izmaylov, J. L. Sonnenberg, D. Williams-Young, F. Ding, F. Lipparini, F. Egidi, J. Goings, B. Peng, A. Petrone, T. Henderson, D. Ranasinghe, V. G. Zakrzewski, J. Gao, N. Rega, G. Zheng, W. Liang, M. Hada, M. Ehara, K. Toyota, R. Fukuda, J. Hasegawa, M. Ishida, T. Nakajima, Y. Honda, O. Kitao, H. Nakai, T. Vreven, K. Throssell, J. A. Montgomery, Jr., J. E. Peralta, F. Ogliaro, M. J. Bearpark, J. J. Heyd, E. N. Brothers, K. N. Kudin, V. N. Staroverov, T. A. Keith, R. Kobayashi, J. Normand, K. Raghavachari, A. P. Rendell, J. C. Burant, S. S. Iyengar, J. Tomasi, M. Cossi, J. M. Millam, M. Klene, C. Adamo, R. Cammi, J. W. Ochterski, R. L. Martin, K. Morokuma, O. Farkas, J. B. Foresman, and D. J. Fox, Gaussian, Inc., Wallingford CT, 2016.

All 3D structure images were made using The PyMOL Molecular Graphics System, Version 2.1.1 Schrödinger, LLC.

B. Validation: simple substituents at $\alpha$ - and $\beta$ - positions 
Before studying the aminoalkyl-substituted acrylamides, we examined reactant and transition state structures for the addition of methane thiolate to a selection of acrylamide substrates with simple substituents at both the $\alpha$ - and $\beta$ - positions. For these structures, the small number of possible conformations were able to be studied systematically. This benchmark is done to verify that the choice of model system and DFT theory are capable of correctly reproducing GSH reactivity trends for acrylamides with modifications at the $\alpha$ - and $\beta$-positions, but can also be used as a baseline for evaluating the various proposed mechanisms in Figs. 3 and 5 in the manuscript. The possible conformers available to the simple acrylamides are $s$-cis and $s$ trans for the reactant, where our model methanethiolate nucleophile can add to the $\beta$ - carbon in either the syn or anti configuration to both the $s$-cis and s-trans conformers. Relative energies and barrier heights are compared to experimentally derived activation barriers in Table $\mathbf{S 1}$.

The structures for the unsubstituted acrylamide 1 found by $\omega$ B97X-D (Fig S1) differ slightly from those reported in our previous work. Structurally, the interaction between the thiolate sulfur and the amide N-H in the s-trans transition structures, as well as the interactions between the methyl $\mathrm{C}-\mathrm{H}$ and the $\alpha$-carbon in the transition structures corresponding to syn addition, are predicted to be stronger by $\omega \mathrm{B} 97 \mathrm{X}-\mathrm{D}$, with intermolecular interaction distances approx. 0.1-0.2 angstroms shorter than in the equivalent B3LYP structures. Both methods produce barrier heights that approximately reproduce the experimental reaction trends across a single choice of transition structure geometry, though we choose to use $\omega$ B97X-D in the present work due to its overall favorable behavior in transition state optimization relative to B3LYP. 

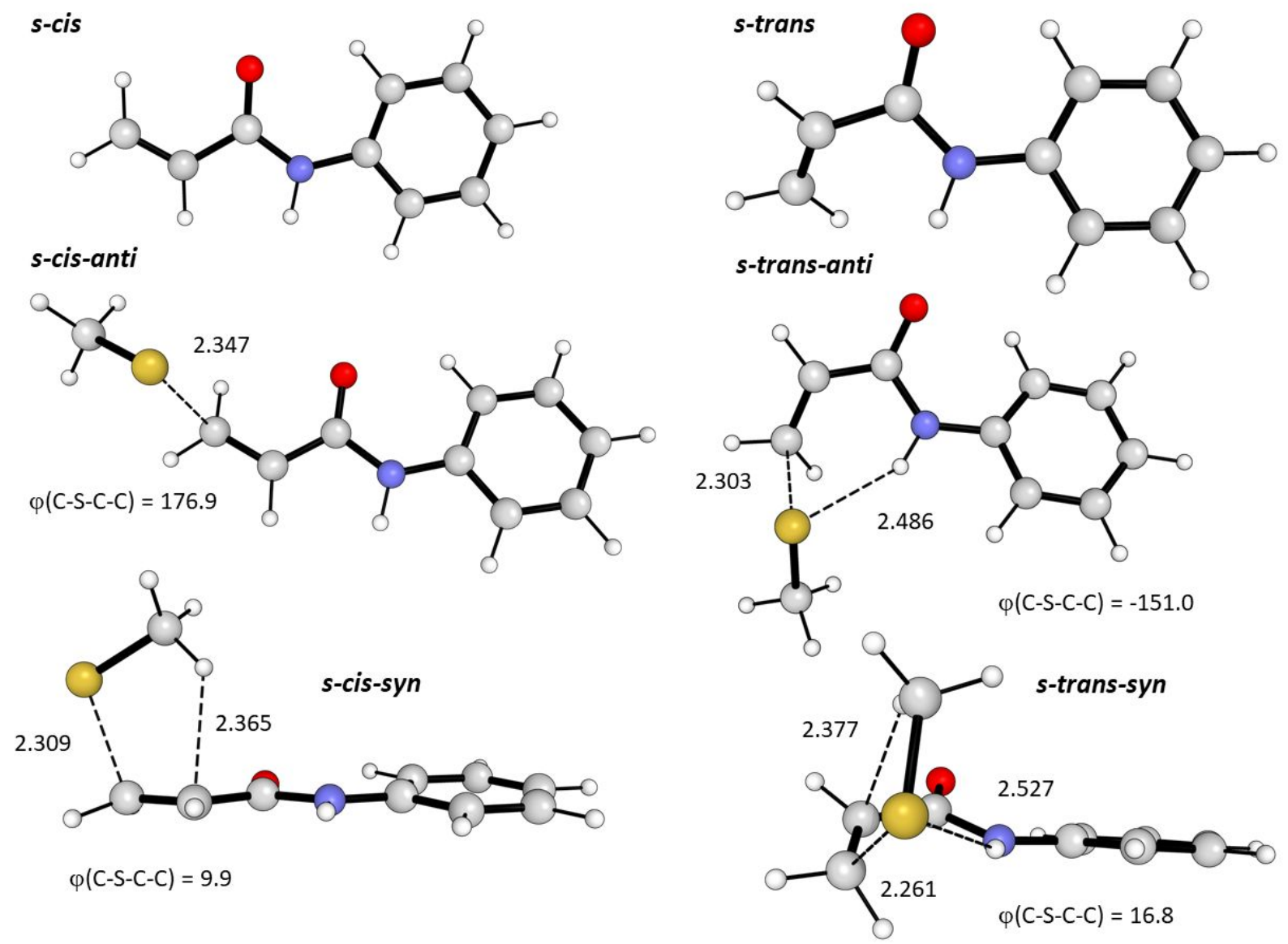

Figure S1. Geometries for the parent N-phenyl acrylamide optimized at the IEFPCM- $\omega$ B97X-D/6$311+G(d, p)$ level of theory. The forming bond and non-covalent interactions are indicated with a dashed line, with distances reported in angstrom. Dihedral angles are reported in degrees.

Table S1. Relative reactant free energies and barrier heights in $\mathrm{kcal} / \mathrm{mol}$. Barriers are computed relative to the global minimum reactant energy. For $\beta-\mathrm{CF} 2 \mathrm{H}$, the three rows correspond to the orientation of the $\mathrm{H}$ atom on the methyl group: 1) in plane with the $\alpha$ carbon $\mathrm{CH}, 2$ ) oriented towards the incoming thiolate, 3 ) oriented away from the incoming thiolate

\begin{tabular}{|c|c|c|c|c|c|c|c|c|}
\hline \multicolumn{4}{|c|}{ Reactants } & \multicolumn{3}{|c|}{ Computed barrier height ${ }^{a}$} & \multicolumn{2}{|r|}{ Experiment } \\
\hline & substituent & $s$-cis & s-trans & s-cis-anti & s-cis-syn & $s$-trans-anti & s-trans-syn & \\
\hline 1 & $\mathrm{H}$ & 0.0 & 2.1 & 21.5 & 19.6 & 20.9 & 19.2 & 24.1 \\
\hline 2 & $\alpha-F$ & 0.0 & 0.5 & 18.8 & 17.6 & 20.3 & 18.9 & $>24.7$ \\
\hline 3 & $\alpha-\mathrm{Cl}$ & 0.0 & 2.4 & 17.6 & 16.1 & 17.0 & 15.7 & 21.6 \\
\hline 4 & $\alpha-\mathrm{CH}_{3}$ & 0.0 & 0.0 & 22.7 & 21.6 & 22.4 & 20.6 & $>24.7$ \\
\hline 9 & $\alpha-\mathrm{CF}_{3}$ & 0.2 & 0.0 & 12.1 & 9.8 & 9.1 & 7.5 & 22.6 \\
\hline 13 & $\beta-\mathrm{CH}_{3}$ & 0.0 & 1.2 & 24.0 & 21.9 & 23.2 & 21.3 & $>24.7$ \\
\hline 14 & $\beta-\mathrm{CF}_{3}$ & 0.0 & 1.3 & 17.9 & 14.8 & 18.0 & 15.1 & 22.6 \\
\hline 15 & $\beta-\mathrm{CF}_{2} \mathrm{H}-1$ & 0.2 & 0.0 & 18.4 & 15.3 & 18.7 & 15.6 & 23.3 \\
\hline
\end{tabular}




\begin{tabular}{lll|llll|}
$\beta-\mathrm{CF}_{2} \mathrm{H}-2$ & 1.0 & 1.1 & 16.5 & 14.3 & 16.3 & 14.0 \\
$\beta-\mathrm{CF}_{2} \mathrm{H}-3$ & 1.0 & 1.1 & 18.6 & 15.7 & 18.2 & 15.4
\end{tabular}

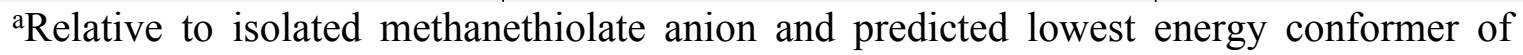
acrylamide substrate.

The model predicts that the $s$-cis reactant geometry is comparable to or lower in energy than the s-trans geometry for all acrylamides. For the transition state, the syn-addition is always lower in energy than the anti- addition, with some acrylamides favoring the s-trans-syn conformer over the s-cis-syn conformer. The correlation to experiment among all of the minimum energy conformers is $\mathrm{R}^{2}=0.14$, which is lower than the correlation across each individual transition state geometry $\left(\mathrm{R}^{2}=0.16-0.27\right)$.

Acrylamide 9 is the largest outlier, with computed barrier heights for all geometries 5-9 $\mathrm{kcal} / \mathrm{mol}$ lower than acrylamide $\mathbf{1 4}$ which was observed to have a similar reactivity (half-lives of 15 and $16 \mathrm{~min}$, respectively). For acrylamides 1, 3, 14 and 15, calculated anti addition barriers to either the $s$-cis or $s$-trans reactant $\left(\mathrm{R}^{2}=0.4\right.$ relative to experiment) are slightly more predictive than syn addition barriers $\left(\mathrm{R}^{2}=0.3\right.$ relative to experiment). The weak correlation to experiment, combined with the observation that higher energy structures improve the correlation to experiment, suggests that there may be an energetic component of reactivity which is neglected by our model.

Acrylamide $\mathbf{1 5}$ has an additional 2 reactant conformers, and 8 transition state conformers resulting from the rotation of the difluoromethyl group. Although structure 15-2, with the $\mathrm{H}$ oriented towards the incoming thiolate, is predicted to have the lowest energy, the barriers predicted by 15-1 and 15-3 are more consistent with the experimental trends (Figure S2). One possible explanation for this observation is that noncovalent, intramolecular interactions like the one between the polar $\mathrm{CF}_{2} \mathrm{H}$ hydrogen and the incoming thiolate, are artificially stabilized by the implicit solvent method due to a lack of proper accounting for potential solvent-solute hydrogen bonding.

This artificial stabilization may also explain the significant error between the calculated and observed barrier heights for acrylamide 9: The $\alpha-C F 3$ group's bulk occludes a strong hydrogen bonding site at the amide $\mathrm{N}-\mathrm{H}$ while in the $s$-cis conformation. The interaction energy of water to this site is approx. $3-5 \mathrm{kcal} / \mathrm{mol}$ to the reactant structures and $2-4 \mathrm{kcal} / \mathrm{mol}$ to the $s$-cis transition state structures across the acrylamides in this test set. For bulky, $\alpha$-substituted acrylamides, the preferred minimum energy structure may therefore be the s-trans conformer in order to maximize the N-H participation in the solvation shell. Similarly, transition structures with the acrylamide in the s-trans orientation may be less favorable than predicted as the sulfur is occupying the N-H site which would be occupied by a water in the s-cis conformation. 

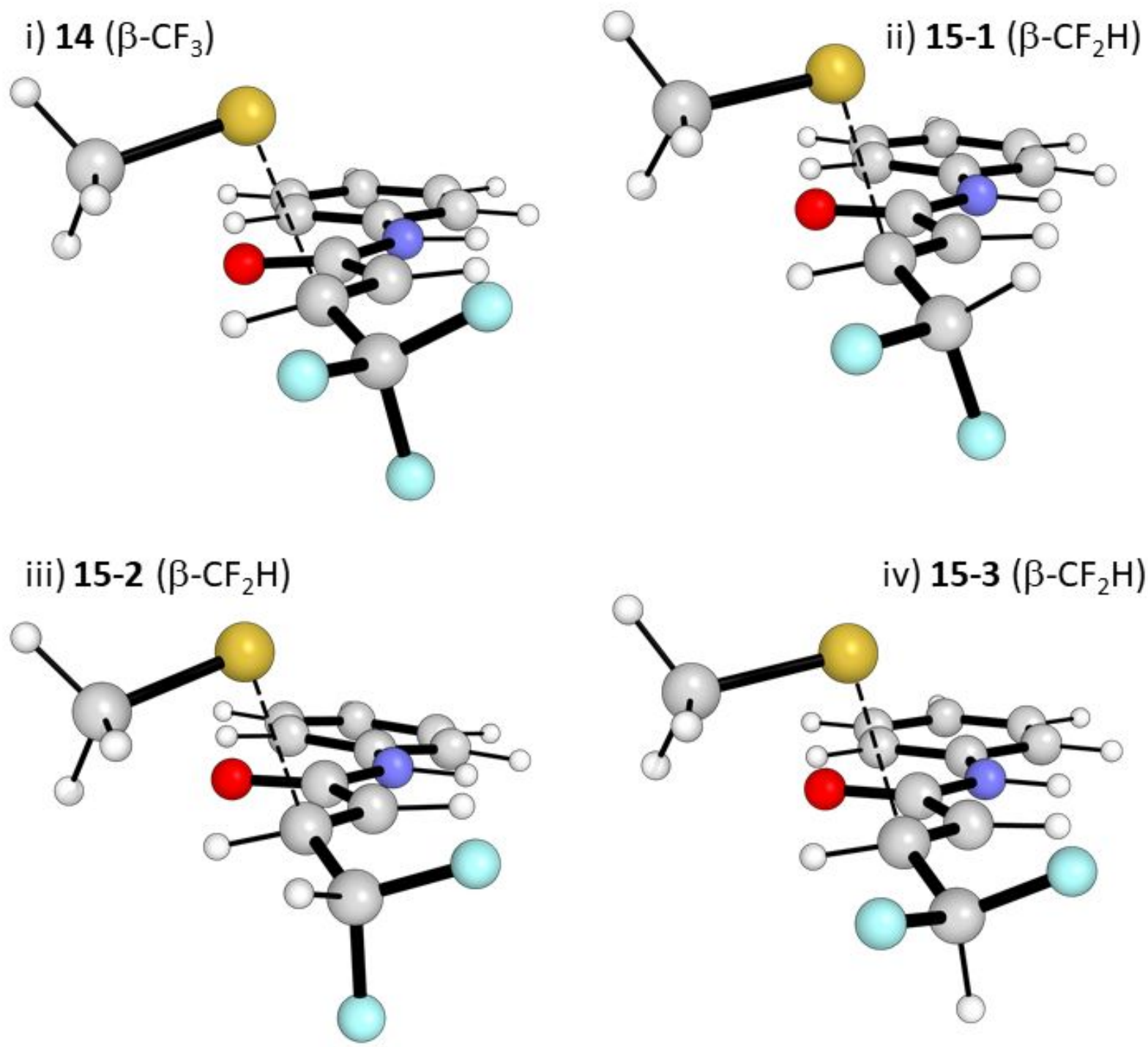

Figure S2. s-cis-anti transition state geometries for acrylamides $\mathbf{1 4}$ and $\mathbf{1 5}$. The 3 conformers of acrylamide 15 differ by the orientation of the $\mathrm{CF}_{2} \mathrm{H}$ group. 15-2 is lower in energy by approximately 2 $\mathrm{kcal} / \mathrm{mol}$, but 15-1 and 15-3's barriers are more consistent with experimental trends, possibly due to the polar C-H in 15-2's inability to participate in the hydration shell, an energetic contribution to reactivity which is absent from implicit solvent calculations.

If we consider only the $s$-cis transition structures (which leave the amide $\mathrm{N}-\mathrm{H}$ accessible to solvent), and use barrier 15-1 instead of 15-2, the correlation for acrylamides 1, 3, 13, and 14 increases from $\mathrm{R}^{2}=0.38$ to $\mathrm{R}^{2}=0.75$ for $s$-cis-anti addition, and from $\mathrm{R}^{2}=0.28$ to $\mathrm{R}^{2}=0.40$ for the s-cis-syn addition. Although it appears as though solute-solvent interactions are important to understanding the relative reactivity of $\alpha$ - and $\beta$ - substituted acrylamides, the implicit solvent DFT barriers are nonetheless able to capture approximate reactive trends when considering conformers which minimize intramolecular non-covalent interactions. These interactions may still contribute to the overall reactivity of the acrylamide moiety in water, but their contribution 
appears to be secondary to the substituent effect on the electrophilicity of the acrylamide substrate.

\section{Water Interaction}

For estimating the strength of solute/solvent hydrogen bonds, [J. Chem. Theory Comput. 2006, 2, 3, 863-872] suggests that it is sufficient to compare the binding energies calculated as the energy of the optimized solute-solvent complex minus the sum of the energies of the optimized solute and solvent monomers. For the purposes of our study, we used the same IEFPCM- $\omega$ B97X-D/6-311+G(d,p) method as described in our manuscript. For the calculation of BSSE energies, the optimized IEFPCM- $\omega$ B97X-D/6-311+G(d,p) complex structures were used as input to a vacuum counterpoise $\omega \mathrm{B} 97 \mathrm{X}-\mathrm{D} / 6-311+\mathrm{G}(\mathrm{d}, \mathrm{p})$ calculation, which is understood to be a reasonable approximation to the BSSE in solvent [Phys. Chem. Chem. Phys., 2007,9, 5555$5560]$.

We carried out water hydrogen-bonding calculations on the acrylamides in Table 4 of the manuscript with a water molecule oriented such that it forms a hydrogen bond with the amide $\mathrm{N}$ $\mathrm{H}$. These calculations were carried out on the s-cis and s-trans reactant structures, as well as the $s$-cis-anti and s-cis-syn transition structures. No additional sampling of the water molecule orientation or the acrylamide geometry was done. Additionally, we also attempted to compute the binding energy water to the $\mathrm{C}-\mathrm{H}$ of the $\mathrm{CF}_{2} \mathrm{H}$ group of acrylamide 15 , which had a hydrogen bond strength of $-1.9 \mathrm{kcal} / \mathrm{mol}$ before BSSE correction. This is approximately equal to the differences in the barrier heights between 15-2 and 15-1/15-3. No transition state for 15 could be located with an explicit water bound to the $\mathrm{C}-\mathrm{H}$ as the water preferred to move to make a stronger hydrogen bond with the accumulated negative charge on the beta carbon.

Table S2 - BSSE corrections and BSSE corrected hydrogen bond energy. All values in kcal/mol.

\begin{tabular}{l|lrrrrrrrr}
\multicolumn{1}{c|}{} & & & \multicolumn{2}{c}{ S-CIS MIN } & \multicolumn{2}{c}{ S-TRANS MIN } & \multicolumn{2}{c}{ S-CIS-ANTI TS } & \multicolumn{2}{c}{ S-CIS-SYN TS } \\
& substituent & bsse & HB energy & bsse & HB energy & bsse & HB energy & bsse & HB energy \\
\hline $\mathbf{1}$ & $\mathrm{H}$ & 1.3 & -4.5 & 1.1 & -4.0 & 1.2 & -3.0 & 1.2 & -3.0 \\
2 & $\alpha-\mathrm{F}$ & 1.1 & -3.6 & 1.2 & -4.6 & 0.9 & -2.8 & 0.9 & -2.9 \\
3 & $\alpha-\mathrm{Cl}$ & 1.1 & -2.8 & 1.1 & -4.5 & 0.9 & -2.1 & 0.9 & -1.9 \\
4 & $\alpha-\mathrm{CH}_{3}$ & 1.0 & -3.4 & 1.1 & -3.7 & 1.0 & -2.2 & 1.0 & -2.3 \\
9 & $\alpha-\mathrm{CF}_{3}$ & 1.2 & -3.0 & 1.2 & -4.6 & 1.2 & -2.1 & 1.2 & -2.6 \\
13 & $\beta-\mathrm{CH}_{3}$ & 1.3 & -4.3 & 1.1 & -4.0 & 1.2 & -2.9 & 1.2 & -2.9 \\
14 & $\beta-\mathrm{CF}_{3}$ & 1.4 & -5.2 & 1.2 & -4.8 & 1.3 & -3.6 & 1.3 & -3.6 \\
15 & $\beta-\mathrm{CF}_{2} \mathrm{H}$ & 1.3 & -4.9 & 1.2 & -4.6 & 1.2 & -3.4 & 1.2 & -3.5
\end{tabular}

Hydrogen bonding availability of the N-H would appear to push the favored conformation of the alpha-substituted acrylamides towards the s-trans geometry in the reactants. The average $\sim 2-3 \mathrm{kcal} / \mathrm{mol}$ hydrogen bonding energy at the transition state is greater than the $\sim 0.5-2 \mathrm{kcal} / \mathrm{mol}$ difference between the s-trans and $s$-cis conformers (table $\mathbf{S 1}$ ), which suggests that the preferred orientation at the transition state would be the $s$-cis conformation. Table $\mathbf{S 3}$ 
shows relative free energies and barrier heights calculated with an explicit water hydrogen bound to the acrylamide $\mathrm{N}-\mathrm{H}$. 
Table S3 - Reactant free energies and free energy barriers relative to the minimum reactant energy for systems with an explicit water bound to the acrylamide N-H. $\mathrm{R}^{2}$ does not include acrylamides 2,4 or 13 . All values are in $\mathrm{kcal} / \mathrm{mol}$.

\begin{tabular}{r|l|rr|rr|r}
\multicolumn{2}{c}{} & \multicolumn{2}{c}{ Reactants } & \multicolumn{2}{c}{ Barrier } & \multicolumn{2}{c}{ Experimental } \\
\hline \multicolumn{2}{r|}{ Substituent } & s-cis & s-trans & s-cis-anti & s-cis-syn \\
\hline $\mathbf{1}$ & $\mathrm{H}$ & 0.0 & 2.7 & 22.9 & 21.2 & 24.1 \\
$\mathbf{2}$ & $\alpha-\mathrm{F}$ & 0.0 & 1.6 & 21.2 & 20.1 & $>24.7$ \\
$\mathbf{3}$ & $\alpha-\mathrm{Cl}$ & 0.9 & 0.0 & 17.8 & 13.8 & 21.6 \\
$\mathbf{4}$ & $\alpha-\mathrm{CH}_{3}$ & 0.7 & 0.0 & 24.8 & 22.7 & $>24.7$ \\
$\mathbf{9}$ & $\alpha-\mathrm{CF}_{3}$ & 4.2 & 0.0 & 17.2 & 16.1 & 22.6 \\
$\mathbf{1 3}$ & $\beta-\mathrm{CH}_{3}$ & 0.0 & 2.3 & 25.9 & 23.4 & $>24.7$ \\
$\mathbf{1 4}$ & $\beta-\mathrm{CF}_{3}$ & 0.0 & 3.9 & 19.0 & 18.7 & 22.6 \\
$\mathbf{1 5}$ & $\beta-\mathrm{CF}_{2} \mathrm{H}$ & 0.0 & 0.9 & 21.6 & 18.4 & 23.3 \\
& & & & & &
\end{tabular}

Acrylamides 9 and $\mathbf{1 4}$ were measured to be approximately equally reactive, but the difference in calculated barrier heights was on the order of 5-6 kcal $/ \mathrm{mol}$ when no explicit waters are used. The addition of a single explicit water reduced the error to $<2 \mathrm{kcal} / \mathrm{mol}$. In the case of the alpha-substituted acrylamides, the introduction of an explicit water either created new interactions, or heavily disturbed the planarity of the s-cis conformer (Fig. S3). These results illustrate how significant of a change that a hybrid explicit-implicit solvation scheme can impose on the geometry of a molecule when small numbers of water molecules are used, and may suggest that a more rigorous/complete treatment of solvation is necessary for the best agreement with experiment across a wide selection of covalent binding warheads.

For the amino-alkyl acrylamides studied in Table $\mathbf{X X}$ of the manuscript, addition of explicit waters in a systematic way is difficult due to the mobility of the amino-alkyl group, and the possibility that a water interacting with the ammonium N-H could also interact with a part of the acrylamide. Instead of investigating the effect of explicit water molecules, we decided to focus on how to best analyze and understand implicit solvent DFT results to limit the errors introduced by the use of implicit solvation. 


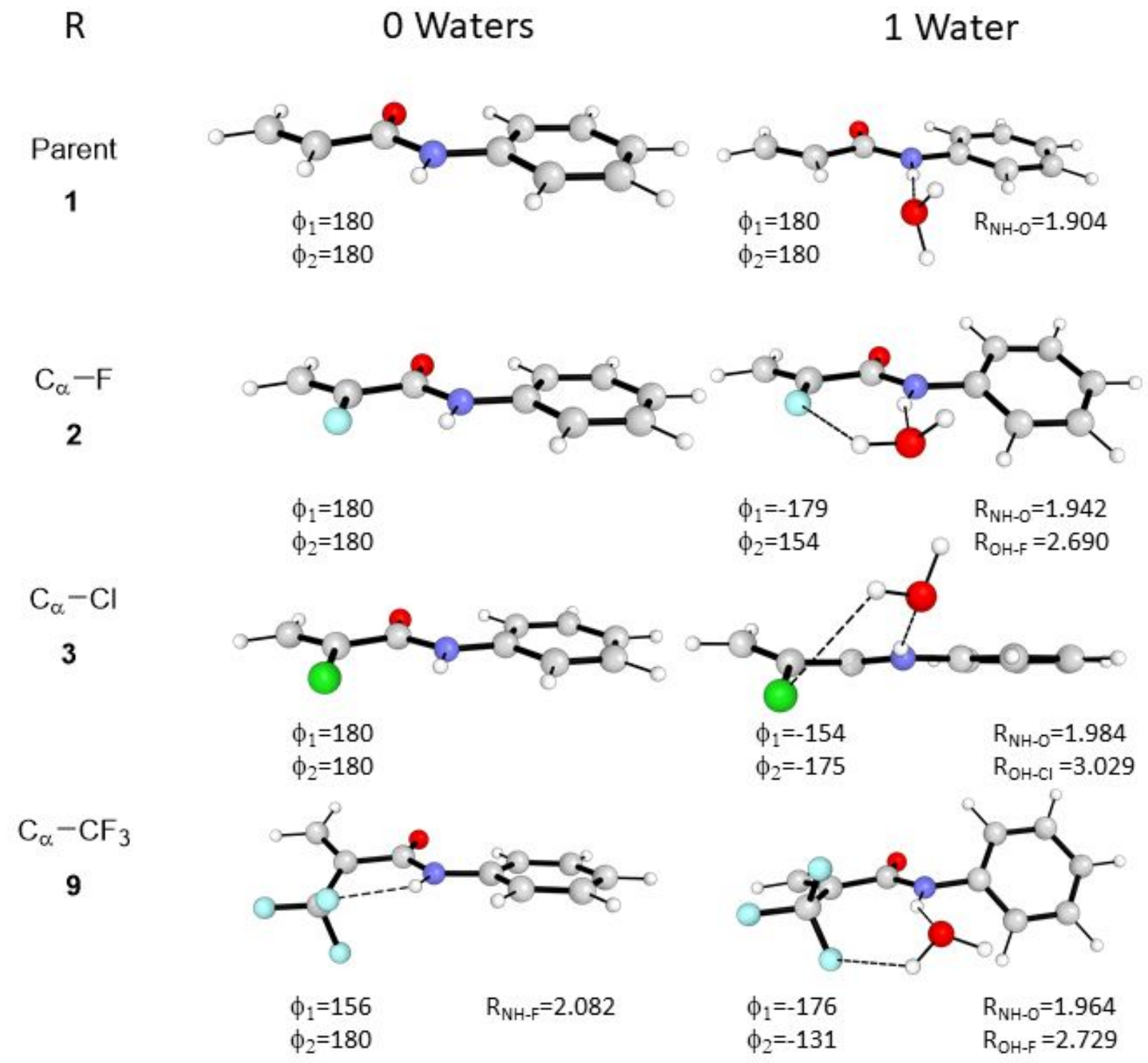

Figure S3 - Selected acrylamide substrates with and without an explicit water. All betasubstituted acrylamides remained flat upon addition of a water similarly to the parent. Noncovalent distances are given in angstroms, dihedral angles are given in degrees. $\phi_{1}=\mathrm{C}_{\beta}-\mathrm{C}_{\alpha}-\mathrm{C}_{\mathrm{O}}-\mathrm{N}$, $\phi_{2}=\mathrm{C}_{\mathrm{O}}-\mathrm{N}-\mathrm{C}-\mathrm{C}$, where $\mathrm{C}_{\mathrm{O}}$ is the ortho carbon nearest to the reader. 


\section{Additional Figures}
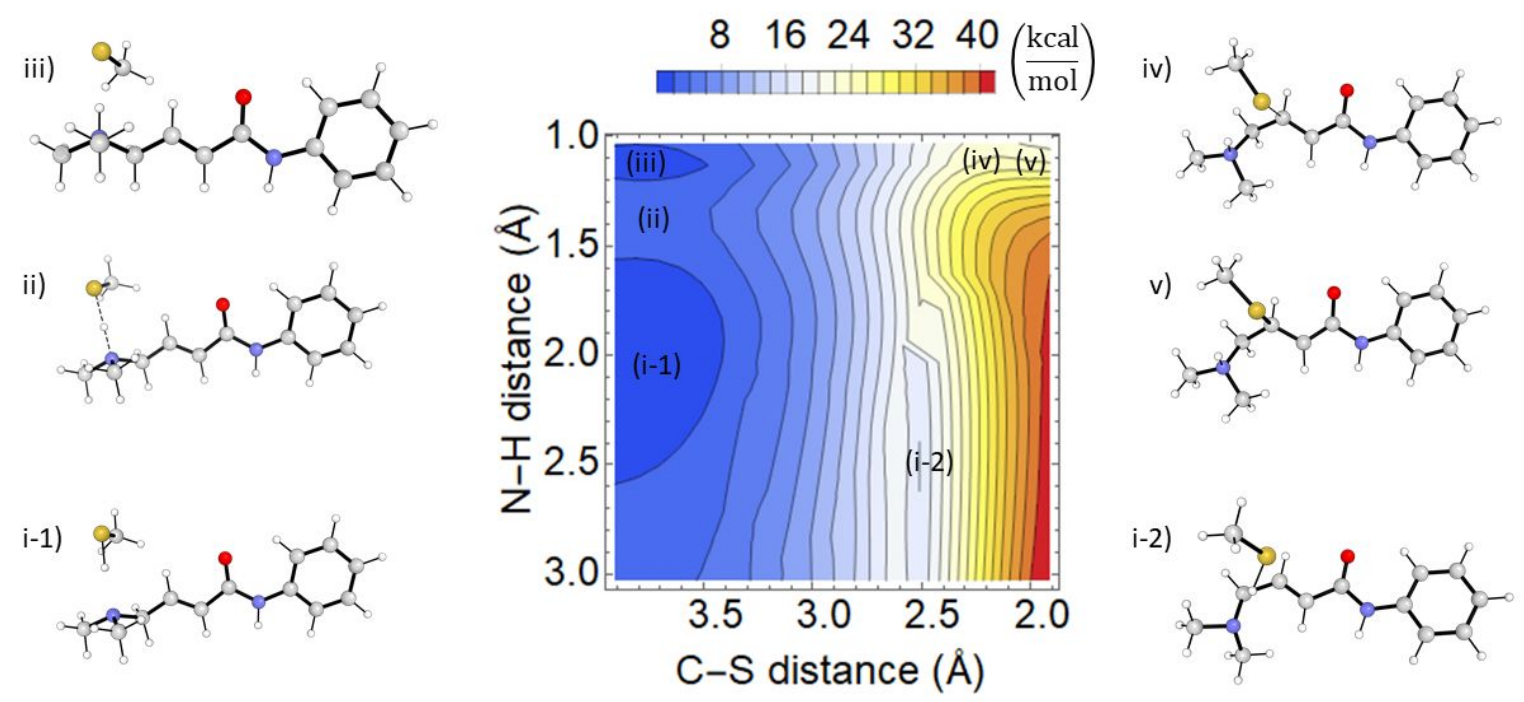

Figure S4 - 2-Dimensional potential energy scan of beta dimethyl ammonia methyl plus methyl thiol, each countour corresponds to $2 \mathrm{kcal} / \mathrm{mol}$. No direct $\mathrm{i}-1$-> v pathway exists, but a clear two step mechanism ( $\mathrm{i}-1>\mathrm{ii}>\mathrm{iii}>\mathrm{iv}>\mathrm{v})$ is observed. An additional high-energy reactant configuration is located at (i-2), corresponding to a bimolecular complex with the sulfur SH oriented between the beta carbon and the ammonia nitrogen, however no barrier is observed directly between reactant minimum i-2 and product $\mathrm{v}$. 